\title{
Placas pleurales por inhalación de fibras de asbesto. Reporte de dos casos y revisión de literatura
}

\author{
DIEMEN DELGADO G.*, ALFONSO MERCADO**, MARÍA DE LOURDES PRECIADO S.*, \\ GABRIEL DÁVALOS P.*** y ASHLEY DELGADO C.****
}

\section{Pleural plaques by inhalation of asbestos fibers. Report of two cases and literature review}

Introduction: Asbestos fiber pleural plaque is characterized by lesions composed of fibrous tissue that are located in the parietal pleura. They usually appear in up to 3 to $58 \%$ of workers who were exposed to asbestos fiber, and 0.5 to $8 \%$ in the general population. The objective of this article is to present two clinical cases of patients who were detected in the chest radiograph pleural alterations associated with exposure to asbestos fibers. Clinical Cases: First case: 49-year-old male patient, building worker with a history of exposure to asbestos fibers. Focal pleural alterations were detected by a chest $x$-ray performed according ILO (International Labour Organization) technique. The presence of pleural plaques was confirmed in a computed tomography of the chest. Second case: Holder, without occupational exposure to asbestos fibers. An anteroposterior chest radiography showed the presence of focal pleural plaque in a CT scan of the chest. Conclusion: Chest x-ray with ILO technique is the basic instrument for the identification of diseases related to asbestos fiber exposure. The study should be completed with a CT scan of the chest whose sensitivity is greater, allowing early detection of pleural abnormalities. Detailed occupational history is essential, as it is the most reliable and practical method to measure asbestos fiber exposure.

Key words: Asbestos; Pleural diseases; occupational exposures; Radiography; Tomography, XRay Computed.

\section{Resumen}

Introducción: Las placas pleurales por fibras de asbesto se caracterizan por lesiones compuestas por tejido fibroso que se ubican en la pleura parietal. Suelen aparecer hasta en un 3\% a 58\% de los trabajadores que estuvieron expuestos a fibras de asbesto, y en un 0,5\% a 8\% en población general. El objetivo de este artículo es presentar dos casos clínicos de pacientes a los que se les detectó en la radiografía de tórax alteraciones pleurales posiblemente asociadas a la exposición a fibras de asbesto. Casos clínicos: Caso 1. Paciente de sexo masculino de 49 años, trabajador de la construcción con antecedentes de exposición a fibras de asbesto. Se le realizó una radiografía de tórax con técnica OIT (Organización Internacional del Trabajo), la que evidenció alteraciones pleurales focales. Una tomografía axial computarizada de tórax, confirmó la presencia de placas pleurales. Caso 2. Paciente de sexo femenino de 79 años, jefa de hogar, sin exposición laboral conocida a fibras de asbesto. En una radiografía de tórax anteroposterior, se observó la presencia de una placa pleural focal. Se solicitó una tomografía axial computarizada de tórax, la que confirmó la presencia de placas pleurales. Conclusión: La radiografía de tórax con técnica OIT es el instrumento básico para la identificación de enfermedades relacionadas con la exposición a fibra de asbesto. El estudio debe ser completado con una tomografía axial computarizada de tórax cuya sensibilidad es mayor permitiendo detectar tempranamente las anomalías pleurales. Es fundamental la historia ocupacional detallada ya que constituye el método más fiable y práctico para medir la exposición a fibra de asbesto.

Palabras clave: Asbesto; Enfermedades pleurales; exposición ocupacional; Radiografí; Tomografía Computarizada.

\footnotetext{
* Docente e Investigador del Departamento de Salud Pública, Universidad de Guadalajara. Guadalajara, México.

** Docente e Investigador de la Facultad de Medicina, Universidad de Texas-Valle del Río Grande. Texas, USA.

*** Docente e Investigador de la Facultad de Medicina, Universidad CEU San Pablo. Madrid, España.

**** Estudiante de Medicina, Universidad Andrés Bello. Viña del Mar, Chile.
} 


\section{Introducción}

Se denomina asbesto a un grupo de minerales compuesto por silicatos hidratados de hierro, sodio y magnesio que se disponen en finas fibras: Está clasificado como un mineral potencialmente carcinógeno para el ser humano por la Agencia Internacional para la Investigación en Cáncer y por la Organización Mundial de la Salud ${ }^{1}$. Se ha documentado ampliamente el daño ocasionado en humanos por la exposición de asbesto, entre ellas: asbestosis, placas pleurales, engrosamiento pleural, derrames pleurales etc., Se calcula que el asbesto es el causante de aproximadamente la mitad de las muertes por cáncer ocupacional en el mundo, siendo ello un importante problema de salud pública ${ }^{2}$.

La fibra de asbesto posee propiedades fisicoquímicas que le confieren un poder aislante térmico y de alta resistencia ${ }^{3}$ a la fricción, lo que ha determinado su utilización en diversos procesos industriales, agrícolas, y de aislamiento en la construcción. Aún existen en muchos países, construcciones, donde se incluyó en la elaboración de las casas materiales en los que predominan las fibras de asbesto ${ }^{4}$.

Otra forma documentada de exposición de este material cancerígeno se encuentra en los familiares de trabajadores ${ }^{5,6}$ que llevaban a sus hogares fibras de asbesto en la ropa de trabajo, zapatos, etc., y en poblaciones cercanas a áreas de explotación del mineral ${ }^{7}$ que se encontraban a varios kilómetros a la redonda con polvos de fibra en suspensión en el aire ${ }^{8}$.

La placa pleural por fibra de asbesto se caracteriza por lesiones compuestas por tejido fibroso que se ubican en la pleura parietal ${ }^{9}$ de predominio en zonas intercostales laterales y posteriores, así como también en pleura mediastínica y diafragmática $^{10}$.

Las lesiones pleuras comúnmente son bilaterales, pero no simétricas y con el tiempo el tejido fibrótico se va coalesciendo y calcificando ${ }^{11}$. Aún no está claro el proceso fisiopatológico del origen de placa pleural por asbesto, pero parece ser que una vez inhalada la fibra de asbesto, por fenómenos físicos de baja resistencia tisular y ayudadas por el gradiente de presión subatmosférico que posee el espacio subpleural ellas, se ubican en la pleura parietal; este fenómeno se puede presentar incluso frente una exposición a baja concentración.

Es de suma importancia reconocer la presencia de placas pleurales secundarias a procesos infecciosos, traumas o cirugías para evitar que nos pueda conducir a errores en la detección de placa pleural por asbesto.
La evidencia ha demostrado que los métodos de visualización de imágenes son útiles como método de pesquisa precoz en poblaciones de alto riesgo, por lo que es de suma importancia el reconocimiento de algunos hallazgos radiológicos característicos en la radiografía de tórax y/o en la tomografía axial computarizada de tórax, ya que en la mayoría de los casos suelen ser un hallazgo radiológico incidental ${ }^{13}$.

La historia ocupacional de exposición a fibras de asbesto y la tomografía axial computarizada de tórax interpretada por un lector con experiencia, constituyen la piedra angular para el diagnóstico de placa pleural por asbesto.

En Chile la legislación vigente desde 2001 prohíbe la utilización de fibra de asbesto en la industria, no obstante, existe activamente la remoción, retiro y manipulación de material con asbesto (techumbre, calderas, ductos etc.,). Las mutualidades y el sistema público de salud son la responsables de la vigilancia en la salud de los trabajadores y de la población en general en especial de los que habitaron o habitan casas elaboradas con fibrocemento.

Esta evaluación se debe realizar regularmente a paciente con antecedentes de haberse desempeñado en lugares con presencia de fibras de asbesto y/o que hayan manipulado materiales con asbesto y que actualmente no estén expuestos. Es por este motivo que a lo menos cada 3 años se le debe realizar a los pacientes expuestos y no expuestos una radiografía de tórax con técnica OIT.

El objetivo de este artículo es presentar dos casos clínicos de pacientes a quienes se les detectó en la radiografía de tórax alteraciones pleurales posiblemente asociadas a la exposición a fibras de asbesto.

\section{Casos clínicos}

\section{Caso 1}

Paciente asintomático de 49 años de edad, de género masculino; trabajador de la construcción y sin antecedentes patológicos, ni toxicológicos importantes. Es evaluado en un programa de salud preventivo en el marco de la Ley 16.744 que hace referencia a la norma chilena sobre accidentes del trabajo y enfermedades profesionales.

Se realiza una radiografía de tórax con técnica OIT donde se detectaron alteraciones pleurales focales (Figura 1) que se originan en la superficie interna de las costillas, extendiéndose a lo largo de los músculos intercostales adyacentes y a nivel del diafragma. 


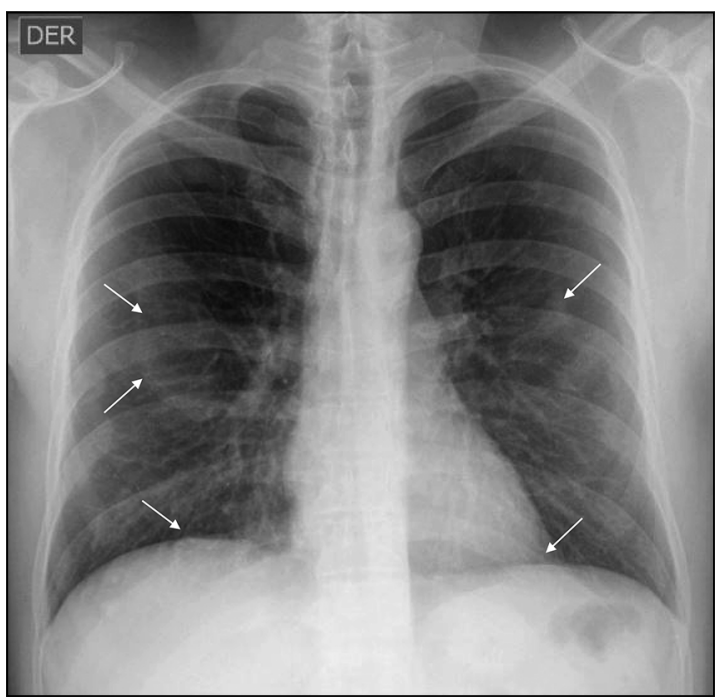

Figura 1. Radiografía de tórax con técnica OIT de paciente con historia de exposición a fibras de asbesto. Se observan múltiples placas pleurales algunas con calcificaciones en su interior.

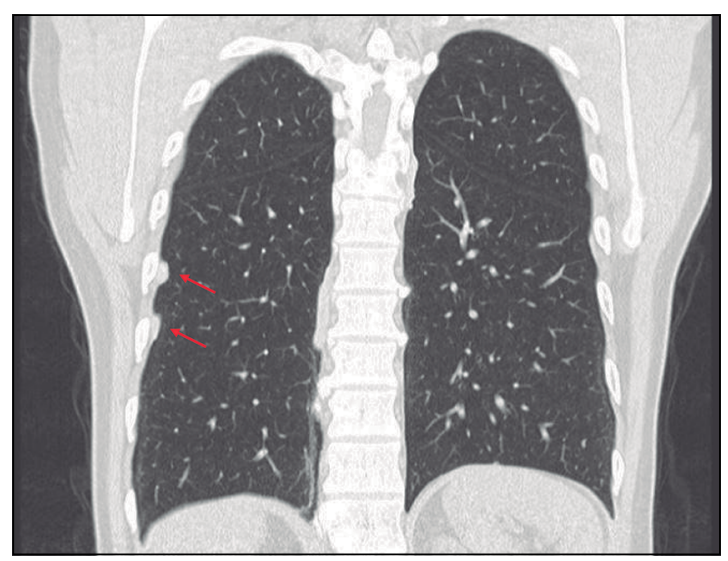

Figura 3. Reconstrucción coronal TC de Tórax. Se observan al menos dos placas pleurales con calcificación en su interior en la pared antero-lateral del hemitórax derecho.

Ante este hallazgo, se efectúa una tomografía axial computarizada de tórax, confirmándose la presencia de placas pleurales calcificadas (Figuras 2,3 y 4$)$.

\section{Caso 2}

Mujer de 79 años de edad, no fumadora; jefa de hogar, hipertensa tratada, que consulta por tos irritativa de tres semanas de evolución. Relata antecedentes de vivir desde los 8 años hasta la actualidad en una población de viviendas sociales construidas en base a fibra de asbesto.

Al examen físico se aprecia paciente afebril

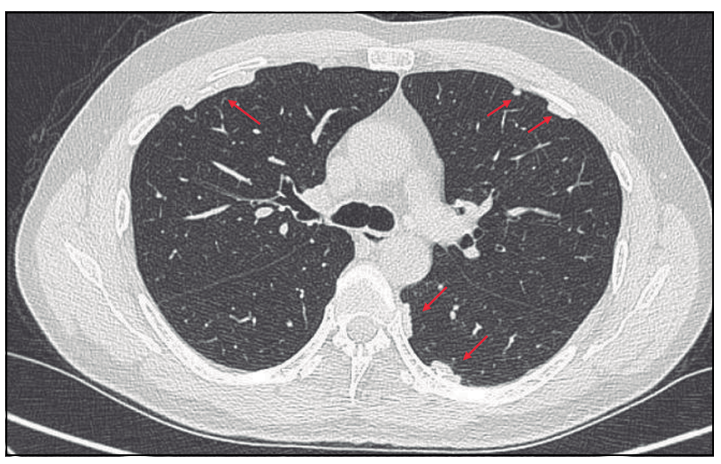

Figura 2. Corte axial de TC de Tórax. Se observan distintas placas pleurales calcificadas en distintos territorios del mismo paciente de la Figura 1.

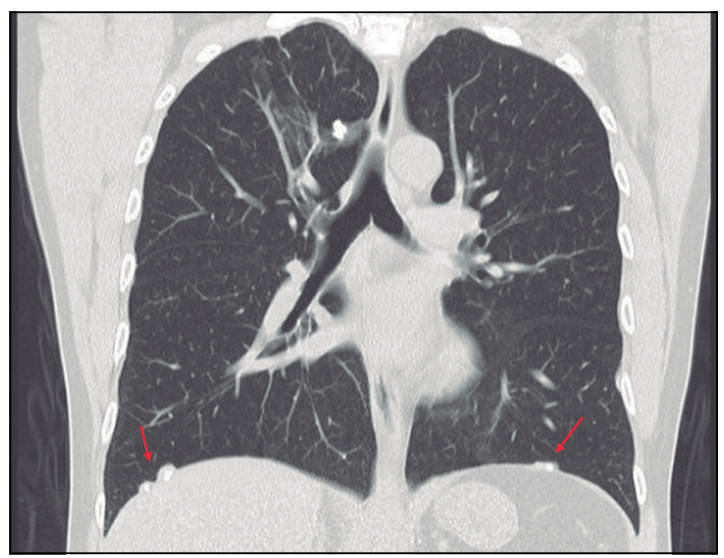

Figura 4. Reconstrucción coronal TC de Tórax. Se observan al menos tres placas pleurales con calcificación en su interior. La ubicación diafragmática es considerada casi patognomónica de la enfermedad. Nótese el respeto de los ángulos costo frénicos, hecho que apoya el diagnóstico de placas pleurales.

y cuyo examen pulmonar es normal. Se efectúa radiografía de tórax que muestra la presencia de una placa pleural en el hemitórax derecho (Figura 5). En la proyección anteroposterior esta placa se ubica en pleura diafragmática observándose coincidentemente una probable imagen pseudonodular parahiliar izquierda.

Dos semanas después la tomografía axial computarizada de tórax confirma la presencia de la lesión pleural, mostrando placas pleurales calcificadas en pared anterolateral del hemitórax izquierdo, cúpula diafragmática izquierda y pleuras paravertebrales Figura 6, 7 y $8 a-b$ ). 


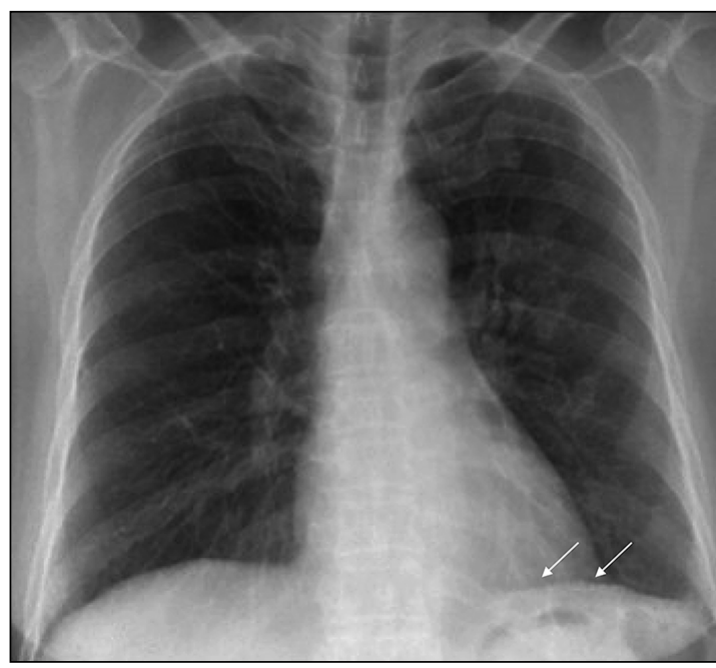

Figura 5. Radiografía de tórax en proyección anteroposterior de paciente de 79 años, sin historia ocupacional de exposición a fibras de asbesto. Se observa con esta técnica placa pleural calcificada en hemidiafragma izquierdo.

\section{Discusión}

En Chile desde enero de 2001 se prohíbe la producción, importación, distribución, venta y uso de fibra de asbesto para la construcción ${ }^{14}$, a partir de esta declaración se pretende limitar la exposición y a más largo plazo eliminar las enfermedades relacionadas con el asbesto, mientras que en otros países han tomado decisiones contra la prohibición total y en favor del uso controlado debido a la alta demanda de fibrocemento en los

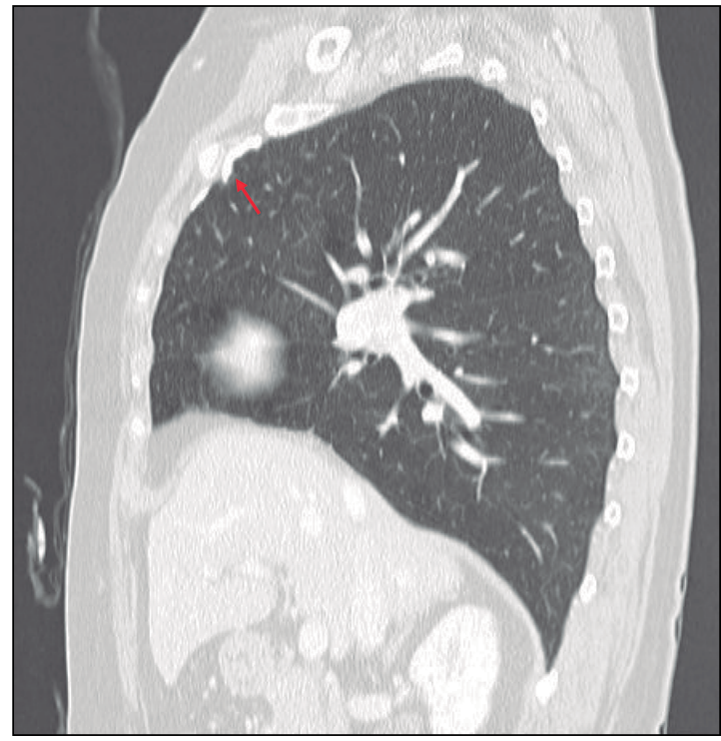

Figura 8a. Reconstrucción sagital de TC de Tórax. Se observa placa pleural calcificada.

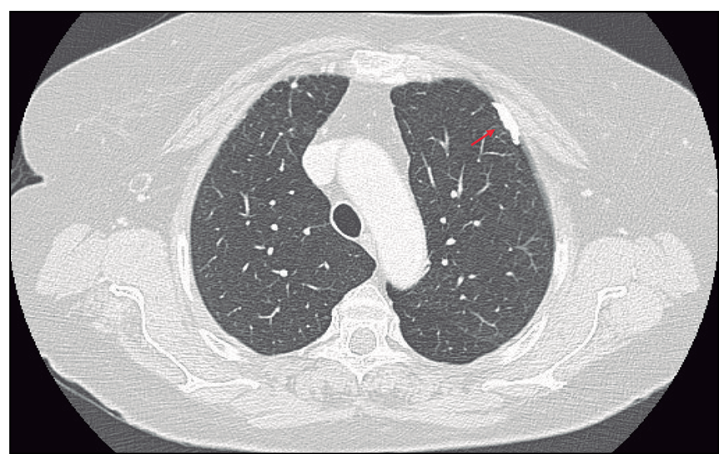

Figura 6. Corte axial de TC de Tórax. Se observa a lo menos una placa pleural con calcificación en su interior en la pared antero lateral del hemitórax izquierdo.

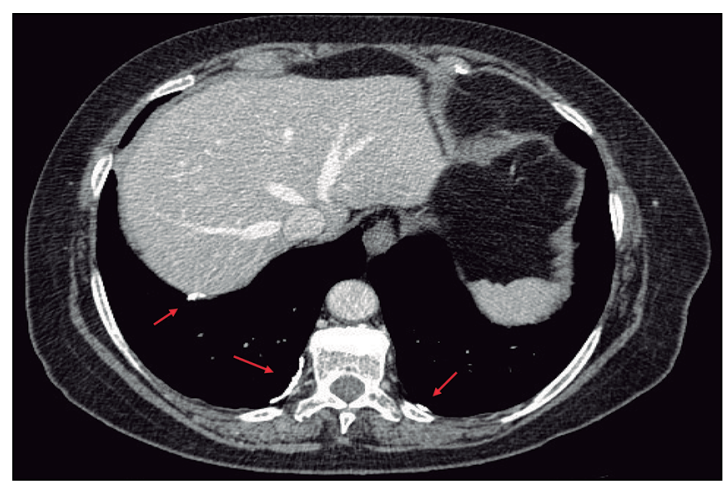

Figura 7. TC de Tórax ventana mediastinal. Se observa placa pleural calcificada en la cúpula diafragmática derecha. Además placas pleurales paravertebrales de predominio derecho calcificadas.

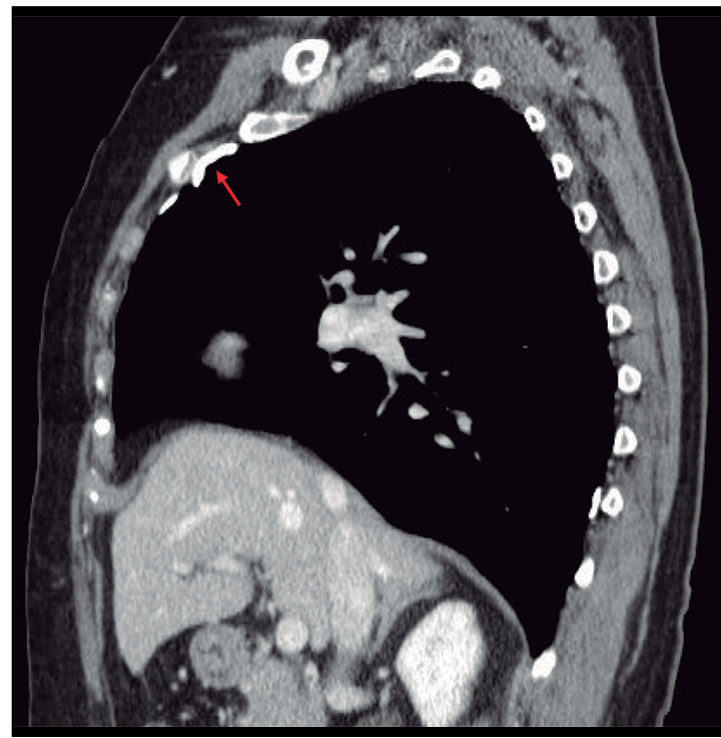

Figura 8b. Reconstrucción sagital de TC de Tórax. Se observa placa pleural calcificada. 
países en desarrollo. Actualmente el $90 \%$ del asbesto mundial proviene de China, India, Rusia y Brasil ${ }^{15}$.

La presencia de placas pleurales asociadas a la exposición a fibra de asbesto suele ser un hallazgo en la tomografía axial computarizada de tórax frecuente en los estudios de imágenes del aparato respiratorio, lo que guarda relación con su prevalencia en la aparición de alteraciones pleurales.

La placa pleural por asbesto debe considerase como un marcador de carga de exposición y su presencia puede aumentar el riesgo de padecer tanto asbestosis como de mesotelioma pleural ${ }^{16}$.

Frente a la presencia de alteraciones tomográficas de la pleura en personas que han estado expuestas a fibra de asbesto es de vital importancia realizar el diagnóstico diferencial. Entre las patologías diferenciales se incluyen:

1. Derrame pleural benigno. Es una manifestación común a la exposición a fibra de asbesto ${ }^{17}$, pudiendo aparecer durante los primeros 20 años tras el primer evento de exposición. La detección se realiza mediante tomografía axial computarizada de tórax observándose un aumento del ancho pleural que se extiende al menos una cuarta parte, es unilateral de predominio izquierdo. El diagnóstico es por exclusión y tras seguimiento de 1 a 3 años ${ }^{18}$, descartando de esta manera el derrame pleural ocasionado por el mesotelioma.

2. Engrosamiento pleural difuso. Se produce a consecuencia de fibrosis de la pleura visceral a menudo bilateral ${ }^{19}$, es marcadamente menos frecuente que las placas pleurales, requiere de una exposición a mayores dosis de fibra de asbesto para su aparición. La detección se realiza mediante tomografía axial computarizada de tórax observándose engrosamiento de bordes irregulares mal definidos con extensión mayor a cuatro espacios intercostales, suele extender a través de las cisuras y es muy raro que las lesiones se calcifiquen. Una de las características del engrosamiento pleural difuso es que produce alteraciones funcionales de tipo restrictivo sin haber compromiso del parénquima pulmonar subyacente ${ }^{20}$.

3. Atelectasias redondas. Es una anomalía infrecuente de la pleura que se invagina sobe sí misma, atrapando el parénquima contiguo, llevando a los bronquios y vasos que van a dicha zona a dibujar una imagen curvilínea que radiológicamente se conoce como cola de cometa ${ }^{21}$. Aunque teóricamente se puede ver en fibrosis pleurales de cualquier origen, en la práctica la mayoría de los casos están asociados a exposición de fibras de asbesto.
En la tomografía axial computarizada de tórax su hallazgo se determina por tres signos radiológicos característicos: una masa redonda u ovalada de entre 2,5 a $7 \mathrm{~cm}$, contactando la superficie pleural, presencia de una estructura lineal en forma de cola en su interior correspondiente con los elementos broncovasculares y engrosamiento de la pleura subyacente ${ }^{22}$. Es importante diferenciar una atelectasia redonda de un carcinoma broncogénico, ya que su aspecto imagenológico puede ser similar.

4. Mesotelioma. Es una neoplasia rara y de mal pronóstico ${ }^{23}$ está asociada en un $80 \%$ con historia de exposición a fibra de asbesto, suele presentarse en pacientes entre 50 a 70 años de edad $^{24}$, siendo más común en hombres con una relación $4: 1$. En la tomografía axial computarizada de tórax se observa un engrosamiento pleural circunferencial, nodular y de más de 1 $\mathrm{cm}$ de espesor. Se puede asociar en un $74 \%$ a derrame pleural unilateral y pérdida de volumen del hemitórax afecto. Su diagnóstico se confirma mediante la obtención de tejido pleural por biopsia preferentemente quirúrgica ${ }^{25}$.

La placa pleural por asbesto presenta una progresión lenta, y no se ha descrito su malignización. Sin embargo, se recomienda su seguimiento como marcador de asbesto ${ }^{26}$.

Clínicamente este tipo de patología no genera síntomas $^{27}$, no afecta la función pulmonar ${ }^{28}$, los hallazgos son radiológicos y la historia ocupacional es fundamental. El conocimiento de la exposición de un paciente a fibra de asbesto nos convierte en un vigilante para detectar otras patologías causadas por dicha exposición que pueden tener repercusión clínica ${ }^{29}$.

\section{Conclusión}

Actualmente la información epidemiológica respecto a lesiones pleurales por asbesto en Chile es muy escasa, probablemente por la limitación en la obtención de información de quienes llevan la vigilancia médica, y por otro lado las dificultades en la recopilación de los registros de defunción por falta de legislación para el desarrollo de autopsias como obligatorias en el país.

La radiografía de tórax es el instrumento básico para la identificación de enfermedades relacionadas con la exposición a fibra de asbestos. La evaluación se realiza en base a la clasificación internacional de la OIT de 2011. Sus limitaciones son la falta de especificidad, así como la limitada sensibilidad para la observación 
de enfermedad pleural por fibra de asbesto, por lo que se hace necesario completar el estudio con una tomografía axial computarizada de tórax cuya sensibilidad es mayor permitiendo detectar tempranamente las anomalías pleurales.

Es fundamental la historia ocupacional detallada ya que constituye el método más fiable y práctico para medir la exposición ocupacional a fibra de asbesto, a través de cuestionarios específicos en los que además de una historia laboral pormenorizada, se obtenga la información acerca del tipo de producto utilizado, duración y métodos de protección utilizados.

El estudio de estos dos casos pretende sensibilizar a la comunidad médica de la necesidad de la evaluación del riesgo de exposición obligatoria a todos los trabajadores que estuvieron expuestos a fibras de asbesto en Chile, específicamente de aquellos que trabajan en demolición, de los que habitan viviendas construidas con material de fibrocemento. Las autoridades de salud pública deben garantizar el derecho de la vigilancia periódica de esta población, incluso prolongándola más allá de la finalización de la exposición dado la demostrada capacidad cancerígena de este mineral, cuya inhalación se ha asociado cáncer de pulmón, laringe y ovario por varios organismos internacionales incluyendo el CDC (Centro para el Control y la Prevención de Enfermedades).

\section{Bibliografía}

1.- KATO K, GEMBA K, ASHIZAWA K, ARAKAWA H, HONDA S, NOGUCHI N, et al. Low-dose chest Computed tomography screening of subjects exposed to asbestos. Eur J Radiol 2018; 101: 124-28.

2.- DOUGLAS T, VAN DEN BORRE L. Asbestos neglect: Why asbestos exposure deserves greater policy attention. Health Policy 2019; 123: 516-19.

3.- DIEGO C, CRUZ M, FERNÁNDEZ R, FERRER J, MARÍN B, MARTÍNEZ C, et al. Recomendaciones sobre el diagnóstico y manejo de la enfermedad pleural y pulmonar por asbesto. Arch Bronconeumol 2017; 53: 437-42.

4.- MAZZEI MA, SARTORELLI P, BAGNACCI G, GENTILI F, SISINNI AG, FAUSTO A, et al. Occupational Lung Diseases: Underreported Diagnosis in Radiological Practice. Semin Ultrasound CT MRI 2018; 40: 36-50.

5.- CHEUNG M, KADARIYA Y, PEI J, TALARCHEK J, FACCIOLO F, VISCA P, et al. An asbestos-exposed family with multiple cases of pleural malignant mesotelioma withhout inheritance of a presisposing BAP1 mutation. Cancer Genetics 2015; 208: 502-7.

6.- KHARAZMI E, CHEN T, FALLAH M, SUNDQUIST
K, SUNDQUIST J, ALBIN M, et al. Familial risk of pleural mesothelioma increased drastically in certain occupations: A natiowide prospective cohort study. Eur J Cancer 2018; 103: 1-6.

7.- TARRÉS J, ABÓS-HERRANDIZ R, ALBERTÍ C, MARTÍNEZ-ARTES X, ROSELL-MURPHY $M$, GARCÍA-ALLAS I, et al. Enfermedad por amianto en una población próxima a una fábrica de fibrocemento. Arch Bronconeumol 2009; 45: 429-34.

8.- VELASCO-GARCÍA MI, RECUERO R, CRUZ MA, PANADES R, MARTÍ G, FERRER J. Prevalencia y distribución del depósito pulmonar de amianto en población urbana española. Arch Bronconeumol 2010; 46: 176-81.

9.- NORBET C, JOSEPH A, ROSSI S, BHALLA S, GUTIÉRREZ F. Asbestos-Related Lung Disease: A Pictorial Review. Current Problems in Diagnostic Radiology 2015; 44: 371-82.

10.- YANG HY, WANG JD, CHEN PC, LEE JJ. Pleural Plaque Related to Asbestos Mining in Taiwan. J Formos Med Assoc 2010; 109: 928-33.

11.- PLAZA C, ÁLVAREZ-SALA R, VILLAMAÑAN E, HERRERO A. Neumoconiosis. Medicine 2018; 12: 3929-35.

12.- KING C, MAYES D, DORSEY D. Benign AsbestosRelated Pleural Disease. Dis Mon 2011; 57: 27-39.

13.- HALLIFAX RJ, TALWAR A, WRIGTSON JM, EDEY A, GLEESON FV. State-of-the-art: Radiological investigation of pleural disease. Respir Med 2017; 124: 88-99.

14.- ACCINELLI R, LÓPEZ L. El asbesto, una epidemia todavía por controlar. Gac Sanit 2017; 31: 365-67.

15.- BARBER C, FISHWICK D. Pneumoconiosis. Medicine 2016; 44 (6): 355-8.

16.- KATO K, GEMBA K, FUJIMOTO N, AOE K, TAKESHIMA Y, INAI K, et al. Pleural irregularities and mediastinal pleural involvement in early stages of malignant pleural mesothelioma and benign asbestos pleural effusion. Eur J Radiol 2016; 85: 1594-600.

17.- VILLENA V, CASES E, FERNÁNDEZ A, DE PABLO A, PÉREZ E, PORCEL JM, et al. Normativa sobre el diagnóstico y tratamiento del derrame pleural. Actualización. Arch Bronconeumol 2014; 50: 235-49.

18.- PORCEL JM. Derrames pleurales benignos persistentes. Rev Clin Esp 2017; 217: 336-41.

19.- LUTHER R. Asbestos-Related Lung Disease. Surg Pathol 2010; 3: 109-27.

20.- AHUJA J, KANNE J, MEYER C. Occupational Lug Disease. Semin Roentgenology 2015; 50: 40-51.

21.- AZOUR L, BILLAH T, SALVATORE M, CHAM M, MENDELSON D, EBER $C$, et al. Clinical Imaging 2018; 50: 250-57.

22.- MARCÍA-SUÁREZ D, SÁNCHEZ-RODRÍGUEZ E, LÓPEZ CALVIÑO B, DIEGO C, POMBAR M. Lowvoltage chest CT: another way to reduce the radiation dose in asbestos-exposed patients. Clin Radiol 2017; 72: 797. e1-797.e10. 
23.- PAAJANEN J, LAAKSONEM S, ILONEN I, WOLFF H, HUSGAFVEL-PURSIAINEN K, KUOSMA E, et al. Computed tomography in the evaluation of malignant pleural mesotelioma-Association of tumor size to a sarcomatoid histology, a more advanced TNM stage and poor survival. Lung Cancer 2018; 116: 73-9.

24.- ODISIO E, MAROM E, SHROFF G, WU C, BENVENISTE AP, TRUONG $\mathrm{M}$, et al. Malignant Pleural Mesothelioma: Diagnosis, Staging, Pitfalls and Follov-up. Semin Ultrasound CT MRI 2017; 38 : 559-70.

25.- RODRÍGUEZ F. Diagnosis and Treatment of Malignant Pleural Mesothelioma. Arch Bronconeumol 2015; 51: 177-84.
26.- CHAMPLIN J, EDWARDS R, PIPAVATH S. Imaging of Occupational Lung Disease. Radiol Clin N Am 2016; 54: 1077-96.

27.- MÁRQUEZ-MARTÍN E, VALENCIA B. Protocolo diagnóstico del engrosamiento pleural. Medicine 2010; 10: 4678-80.

28.- CLARK K, FLYNN J, KARMAUS W, MOHR L. The Effects of Pleural Plaques on Longitudinal Lung Function in Vermiculite Miners of Libby, Montana. Am J Med Sci 2017; 353: 533-42.

29.- BARNIKEL M, MILLION PM, KNOOP H, BEHR J. The natural course of Lung Function decline in asbestos exposed subjects with pleural plaques and asbestosis. Respir Med 2019; 154: 82-5.

Correspondencia a:

Dr. Diemen Delgado García

Docente de Medicina del Trabajo de la Universidad San

Sebastián.

Santiago, Chile.

Email: diemenddg@hotmail.com 